\title{
Study of Adhesion Interaction Using Atomic Force Microscopy
}

\author{
J. Gryboś, G. Pyka-Fościak, K. Lebed, M. LekKa*, \\ Z. Stachura and J. STYCZEŃ
}

The Henryk Niewodniczański Institute of Nuclear Physics Polish Academy of Sciences, Radzikowskiego 152, 32-341 Cracow, Poland

\author{
(Received April 26, 2004)
}

\begin{abstract}
An atomic force microscope is a useful tool to study the interaction forces at molecular level. In particular the atomic force microscope can measure an unbinding force needed to separate the two single molecule complexes. Recent studies have shown that such unbinding force depends linearly on the logarithm of the applied loading rate, defined as a product of scanning velocity and the spring constant characterizing the investigated system (cantilever vs. surface). This dependence can be used to study the energy landscape shape of a molecular complex by the estimation of energy barrier locations and the related dissociation rates. In the present work the complex consisting of ethylene(di)aminetetraacetic acid and the bovine serum albumin was measured. The dependence between the unbinding force and the logarithm of the loading rate was linear. Using the Bell model describing the dissociation of the above molecules caused by the action of the external bond breaking force, two parameters were estimated: the dissociation rate and the position of the energy barrier needed to overcome during a transition from a bound to unbound state. The obtained results are similar to those obtained for a typical ligand-receptor interaction.
\end{abstract}

PACS numbers: 87.64.Dz, 68.35.Gy

\section{Introduction}

The knowledge about the mechanism and strength of the chemical binding forces is important for the understanding of the variety range of phenomena, like for example the adhesion of the polymers to different types of substrates, friction, or

*corresponding author; e-mail: Malgorzata.Lekka@ifj.edu.pl 
molecular interactions between cells. Particularly, the mechanism of the adhesion between biomolecules is crucial for the diversity of the biotechnological applications like bioseparation, immunoadsorption, different types of cell-to-cell interactions, blood clotting or even oncogenic transformation [1]. The formation of an adhesion contact between two molecules is a special case of processes in which the formation and dissociation of chemical compounds occur. Therefore, it could be described as a dynamic process in which a certain number of bonds is created and some bonds, in the same time, are broken. Thus, the association and dissociation rates express the speed of formation and breaking bonds at given conditions because usually the chemical reaction depends on many factors as temperature or solvent properties $[2,3]$.

One of the methods that can be applied in the investigations of the dissociation process can be the atomic force microscopy (AFM). In recent years, this method has become a very useful technique to investigate many biological problems, ranging from high-resolution structural images of biological objects [4] to the strength of the molecular interaction [5]. These latter applications are possible due to a very high force resolution extending down to picoNewtons range, which allows detecting a force of a single molecular pair, like for example between two proteins [6] or two functional chemical groups [7]. Usually, the AFM probe (i.e. the cantilever with a sharp tip) is functionalized with one type of molecules while the other, complementary molecules are immobilized on a substrate [8]. When the tip is in a close proximity of the surface (or when it touches the surface), the cantilever is deflected due to the presence of different interactions (i.e. van der Waals, magnetic or electrostatic forces). The deflection can be recorded as a function of a relative sample position and can be converted into a force value. The force needed to separate the AFM tip from the surface corresponds to the unbinding force.

The idea of the measurements of the unbinding force was mainly applied to the characterization of ligand-receptor interaction occurring between different pairs of molecules. As a very sensitive force probe, the AFM has been used to determine the unbinding force between streptavidin-biotin molecules [9] as well as those between monoclonal antiferritin and AFM probes functionalized by ferritin [10]. Also, the interactions occurring between complementary strands of DNA oligomers were studied [3].

The dynamics of the dissociation process between biomolecules was for the first time described by Bell [11]. Bell stated that the application of the externally applied force $f$ separating the ligand from the receptor causes an increase in dissociation rate $k_{\mathrm{r}}(f)$. The proposed increase followed the exponential rule

$$
k_{\mathrm{r}}(f)=k_{\mathrm{r}}^{0} \exp \left(r_{0} f / k_{\mathrm{B}} T\right)
$$

where $k_{\mathrm{r}}(f)$ - dissociation rate in the presence of the force $f$ that breaks the individual bonds (i.e. an unbinding force), $k_{\mathrm{r}}^{0}$ - dissociation rate in the absence 
of the force $f, r_{0}$ - position of the energy barrier that should be overcome during the unbinding process, $k_{\mathrm{B}}$ - the Boltzman constant, $T$ - temperature.

The probability of breaking of a single bond in a certain time interval is a function of the force that breaks the bond. The maximum of the probability distribution corresponds to the most probable value of the force $f^{*}$ that breaks the bond and it can be attributed to the unbinding force determined from the AFM measurements. The dissociation rate can be evaluated when the unbinding force is linearly applied in time (the loading rate $r_{f}$ is constant). Thus, the relationship between $f^{*}$ and $\ln \left(r_{f}\right)$ can be expressed as follows $[12,13]$ :

$$
f^{*}=\frac{k_{\mathrm{B}} T}{r_{0}} \ln \left(r_{f}\right)+\frac{k_{\mathrm{B}} T}{r_{0}} \ln \left(\frac{r_{0}}{k_{\mathrm{r}}^{0} k_{\mathrm{B}} T}\right),
$$

where the loading rate $r_{f}$ is a product of the scanner velocity $v$ and the cantilever spring constant $k$. Therefore, by performing the measurements at different values of the loading rates more information on the molecular interaction potential can be obtained $[14,15]$.

The relation between $f^{*}$ and $\ln \left(r_{f}\right)$ is linear and from its slope $b$ and the intercept $a$ one can calculate $r_{0}$ and $k_{\mathrm{r}}^{0}$ according to the relationships

$$
\begin{aligned}
& k_{\mathrm{r}}^{0}=\frac{1}{b} e^{-a / b}, \\
& r_{0}=\frac{k_{\mathrm{B}} T}{b} .
\end{aligned}
$$

The errors may be estimated in a standard way

$$
\begin{aligned}
& \Delta r_{0}=\left|\frac{k_{\mathrm{B}} T}{b^{2}}\right| \Delta b, \\
& \Delta k_{\mathrm{r}}^{0}=\sqrt{\left(\frac{\partial k_{\mathrm{r}}^{0}}{\partial a} \Delta b\right)^{2}+\left(\frac{\partial k_{\mathrm{r}}^{0}}{\partial b} \Delta a\right)^{2}} .
\end{aligned}
$$

In the present study, the atomic force microscopy was applied to study the interaction forces between two types of molecules: bovine serum albumin and ethylene(di)aminetetraacetic acid. The linear dependence between the unbinding force and the logarithm of the loading rate was obtained indicating that this mode of the AFM operation could be applied not only for the studies of typical ligand-receptor complexes but also in the investigations of the unbinding process between molecules that interact involving non-specific forces. On a basis of the force distributions, the most probable unbinding force was evaluated. From the relation between the unbinding force and the logarithm of the loading rate, the dissociation rate and the position of the energy barrier were calculated. 


\section{Materials and experimental method}

\subsection{Modification of the AFM cantilevers}

The standard silicon nitride cantilevers (Park Scientific Company, Atos GmbH, Germany) with pyramidal shaped tips were used. The cantilevers were covered with the ethylene(di)aminetetraacetic acid (EDTA, Fluka) using the reaction scheme for the functionalization of the tip presented in [16]. First, the AFM tips were washed with acetone for five minutes and exposed to UV for 20 minutes to remove organic contaminants. After irradiation, the cantilevers were incubated for two hours in $4 \%(\mathrm{v} / \mathrm{v})$ solution of the triaminopropyltriethoxysilane (APTES,

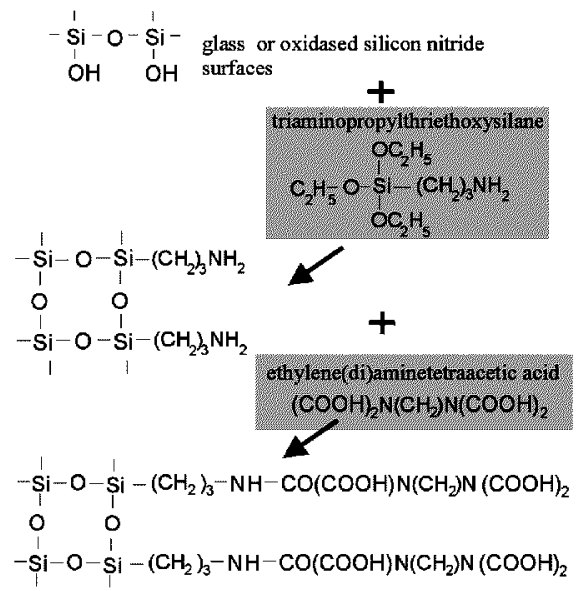

Fig. 1. One of the possible scheme of the glass coverslip functionalization with the ethylene(di)aminetetraacetic acid.

Sigma) in a toluene in order to enrich the tip surface with amino groups. Next, they were washed in alcohol once and three times in phosphate buffer saline (PBS, $\mathrm{pH}=7.4$, containing $0.01 \mathrm{M} \mathrm{H}_{3} \mathrm{PO}_{4}, 0.0027 \mathrm{M} \mathrm{KCl}$, and $0.137 \mathrm{M} \mathrm{NaCl}$, Sigma). The last step was the immersion of the cantilevers in $0.1 \mathrm{mg} / \mathrm{ml}$ solution of the EDTA for one hour. They were stored in a fridge for 24 hours at the temperature of $2^{\circ} \mathrm{C}$ before use. One molecule of the EDTA consists of five carboxyl groups which in water solution become negative charged and therefore the molecule binds to the surface amino groups. The possible reaction scheme is presented in Fig. 1.

\subsection{Protein immobilization on a glass substrate}

The immobilization of the albumin (BSA, bovine serum albumin, Sigma) onto glass surface followed the procedure described in [16]. The glass coverslips were pretreated in the same way as the silicon nitride tips were treated until the step of silanization with the APTES solution. After washing in alcohol once and 
three times in the PBS, the coverslips were immersed into $2.5 \%$ glutaraldehyde (Fluka) solution in PBS for 30 minutes. Next, the coverslips were immersed in $0.5 \mathrm{mg} / \mathrm{ml} \mathrm{BSA}$ water solution for one hour. Such prepared substrates with protein were stored in the PBS in a fridge at the temperature of $2^{\circ} \mathrm{C}$ for 24 hours before use.

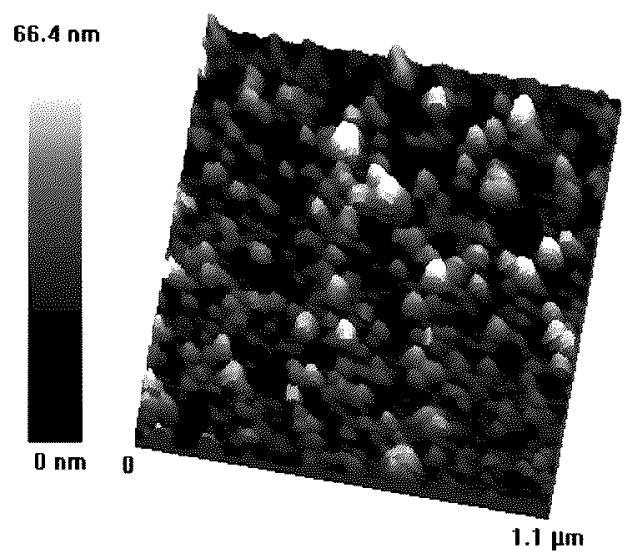

Fig. 2. The AFM image of the albumin immobilized onto glass coverslip. The determined indentation depth $(\sim 50 \mathrm{~nm})$ suggested that the protein layer was thick (at $\mathrm{pH}=7$ the shape of albumin is an ellipsoidal with two axes of $4 \mathrm{~nm}$ and $14 \mathrm{~nm}$ ).

The bovine serum albumin (molecular weight $69 \mathrm{kDa}$ ) was chosen due to its affinity to many types of ligands like bilirubin or hematin. Particularly, albumin has a great ability for binding small negatively charged molecules as fatty acids $[17,18]$. Figure 2 presents the image of the topography of albumin molecules immobilized on the glass substrate using the AFM tips coated with the EDTA molecules.

\subsection{Atomic force microscopy}

Force measurements were carried out using the AFM working in contact mode, equipped with a "liquid cell" setup [19]. All measurements were performed in deionized water (Cobrabid purification system, $0.06 \mu \mathrm{S}$ ) adjusted to $\mathrm{pH}=7.0 \mathrm{using}$ $\mathrm{NaOH}$ base, at the room temperature. Force versus distance curves were repeatedly measured at various surface locations. On each sample 4-7 positions were chosen and at each position 100 to 200 force curves were recorded. The spring constants of AFM cantilevers were: $0.01,0.03,0.05$, and $0.1 \mathrm{~N} / \mathrm{m}$. The obtained system spring constants (taking into account both the cantilever and the substrate) were in the range of $5-10 \mathrm{mN} / \mathrm{m}$ (estimated from the slope of the force versus displacement relationship of the retraction curve, [5]). The range of the scanner velocities of the approach-retract cycles varied from 1 to $8 \mu \mathrm{m} / \mathrm{s}$. By using various cantilever spring constants and several velocities it was possible to produce different values of the loading rate $r_{f}$. The loading rate $r_{f}$ was defined above as a product of the 


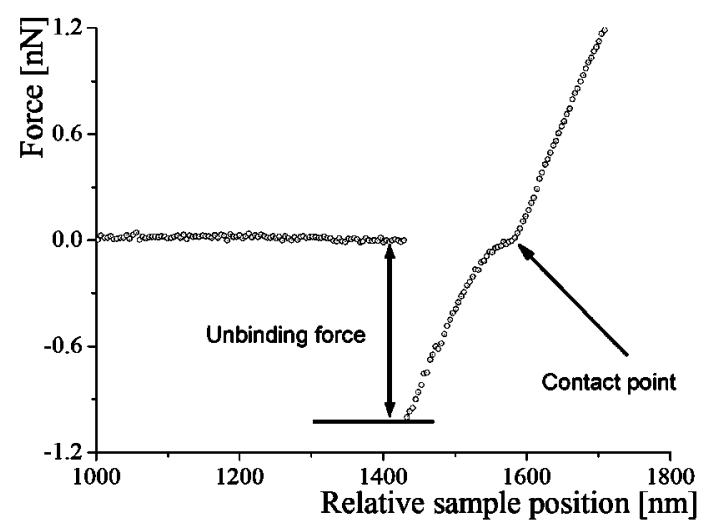

Fig. 3. The typical force distance curve measured by AFM - only a retracting part is shown.

scanner velocity and the system spring constant. The unbinding force values were obtained from the analysis of the retracting part of force-versus-distance curves. The typical force-distance curve measured by the AFM is shown in Fig. 3.

\section{Results and discussion}

The interaction between albumin immobilized on a flat surface and the ethylene(di)aminetetraacetic acid molecules attached to the AFM tip can be explained involving the ionic bonds occurring between the albumin's amino and EDTA's carboxyl groups as they are both charged in water solution. The bond strength was determined from the force distribution delivered by the AFM measurements of the unbinding force. For each loading rate the corresponding force distribution was created. For instance, two histograms taken for the rate of $50 \mathrm{nN} / \mathrm{s}$ are presented in Fig. 4. They were created with the bin size of $50 \mathrm{pN}$, significantly larger that the noise fluctuations of the base line (the part of curve, where there is no contact between the tip and surface). The maximum of the histogram was attributed to the force breaking a single bond. The maximum values were $227 \pm 7 \mathrm{pN}$ for BSA-EDTA complex measured in pure water (Fig. 4A) and $311 \pm 10 \mathrm{pN}$ when solution contained $1 \mathrm{mM}$ EDTA (Fig. 4B). The errors were estimated by the division of the standard deviation by the square root of the number of the adhesion events. It should be mentioned that the pull-off force measured using the AFM gives overall force acting between two surfaces - both specific (that interact only within the contact area), and non-specific ones (acting in large distances like for example electrostatic forces). Thus, in the case of non-complementary complexes, the influence of the environment properties could be observed. In this manner, the change in the positions of the distribution maximum might be attributed to the influence of the free EDTA molecules. 

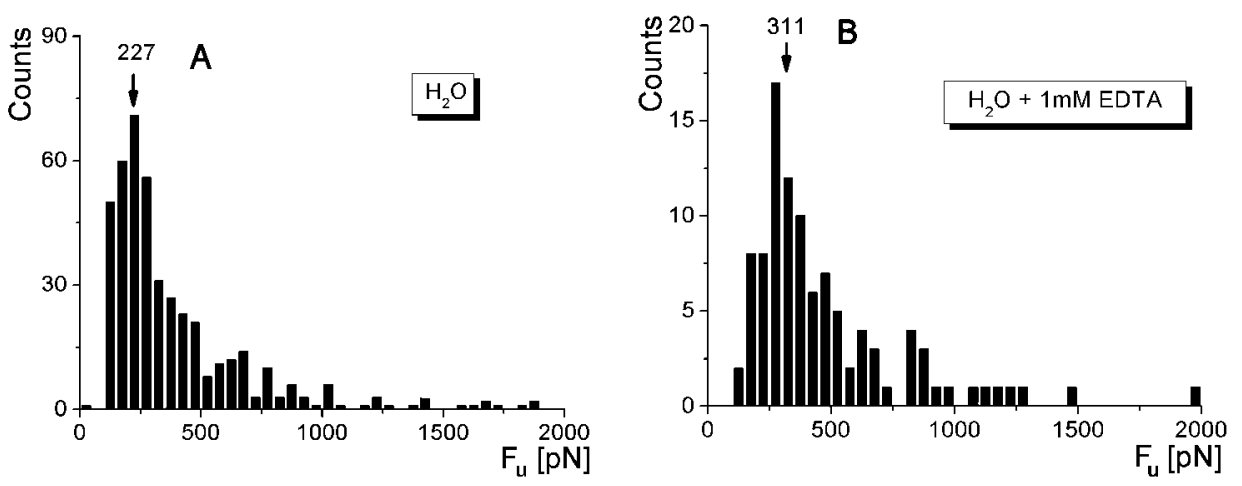

Fig. 4. Examples of frequency distributions of the unbinding force measured for particular loading rate $(50 \mathrm{nN} / \mathrm{s})$ between the EDTA-BSA molecules in pure water (A) and in the presence of $1 \mathrm{mM}$ EDTA aqueous solution (B).

In order to confirm that the measured unbinding force originated from the interaction between albumin and ethylene(di)aminetetraacetic acid, the fraction of curves with the observed adhesion was estimated. As the EDTA molecules were dissolved in water and as they could cover the albumin surface, the recorded curves should show less adhesion events than in the case of pure water. The observed fraction $72.6 \%$ (water) decreased to $45.7 \%$ after adding $1 \mathrm{mM}$ concentration of EDTA.

The dynamic AFM measurements allowed the determination of the parameters that characterize the bond formation and dissociation of the interacting molecules. According to the transition state theory, the dissociation occurring between the ligand-receptor pair can proceed via an intermediate state which can be confirmed by the presence of two (or more) linear regions in the dependence between the unbinding force and the logarithm of loading rate [20-22]. The obtained for BSA-EDTA complex dependence between the unbinding force and the logarithm of the loading rate showed two linear regions (marked as (a) and (b)) presented in Fig. 5. Both regions were fitted with the linear regression according to Eq. (2). In the region (a) the loading rate varied from 5 to $30 \mathrm{nN} / \mathrm{s}$ and in the region (b) from 30 to $130 \mathrm{nN} / \mathrm{s}$. The calculated slopes were $49 \mathrm{pN}$ and $232 \mathrm{pN}$ for regions (a) and (b), respectively.

These results indicate that the dissociation process between the EDTA and the BSA molecules follow the interaction similar to that observed in ligand-receptor complex involving transition through the intermediate state [14]. The calculated parameters describing the dissociation process, the dissociation rates $k_{\mathrm{r}}^{0}$, and the position of the energy barrier $r_{0}$, were $6.4 \mathrm{~s}^{-1}, 0.84 \AA$ for region (a) and $49.1 \mathrm{~s}^{-1}$, $0.20 \AA$ for region (b) (cf. Table I). The region of higher loading rates (b) may be attributed to the transition from the bound state to the intermediate state and the region of smaller rates (a) - to the transition between the intermediate to an unbound state. The results indicated that the dissociation process was described 


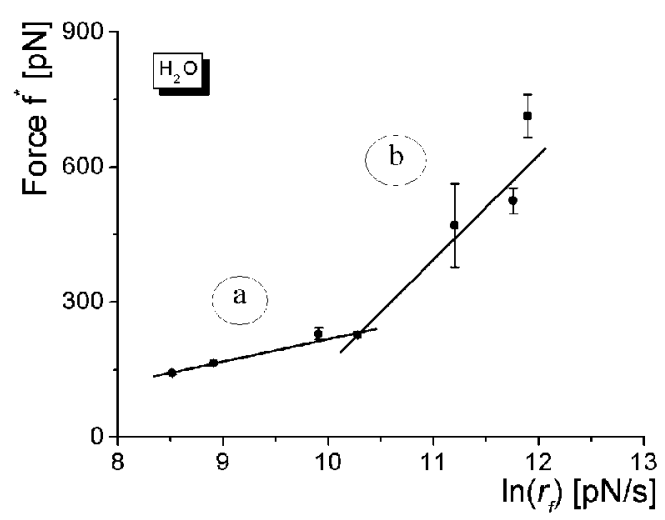

Fig. 5. The most probable unbinding force $f^{*}$ as a function of the logarithm of the loading rate. Two different linear regions are marked as (a) and (b). The measurements were performed for the EDTA-BSA pair in water.

TABLE I

Parameters describing the dissociation process calculated for EDTA-BSA pair on the basis of the Bell model. The measurements were performed in deionized water.

\begin{tabular}{c|c|c|c|c}
\hline \hline Loading rate range $[\mathrm{nN} / \mathrm{s}]$ & Intercept $[\mathrm{pN}]$ & Slope $[\mathrm{pN}]$ & $r_{0}[\AA]$ & $k_{\mathrm{r}}^{0}\left[\mathrm{~s}^{-1}\right]$ \\
\hline Linear region a: $5-30$ & $-282 \pm 38$ & $49 \pm 4$ & $0.84 \pm 0.07$ & $6.4 \pm 3.5$ \\
Linear region b: $30-130$ & $-2167 \pm 175$ & $232 \pm 16$ & $0.20 \pm 0.01$ & $49.1 \pm 35.0$
\end{tabular}

by the molecular potential composed of two barriers, the inner and the outer one (which means that the dissociation should overcome two transitions).

Knowing that the interaction between the albumin and ethylene(di)aminetetraacetic acid molecules is not a specific one, the effect of the free EDTA molecules (added to water) on the binding ability of the BSA was studied. The same calculations of the dissociation parameters were performed for two cases, when the added EDTA concentration was $1 \mathrm{mM}$ and $1 \mu \mathrm{M}$. Results are presented in Fig. 6.

Independently of concentration of the EDTA molecules in water, each case showed only one linear region with a different set of parameters (Table II). This means that the dissociation process overcame only through one energy barrier

TABLE II

Dissociation parameters calculated according to the Bell model for EDTA-BSA pair measured for two concentrations: $1 \mu \mathrm{M}$ and $1 \mathrm{mM}$ aqueous solution of EDTA for the loading rate range of $20-70 \mathrm{nN} / \mathrm{s}$.

\begin{tabular}{c|c|c|c|c}
\hline \hline Concentration $[\mathrm{mM}]$ & Intercept $[\mathrm{pN}]$ & Slope $[\mathrm{pN}]$ & $r_{0}[\AA]$ & $k_{\mathrm{r}}^{0}\left[\mathrm{~s}^{-1}\right]$ \\
\hline 0.001 & $270 \pm 17$ & $-2053 \pm 185$ & $0.15 \pm 0.01$ & $39.3 \pm 25.4$ \\
1 & $145 \pm 7$ & $-1282 \pm 84$ & $0.28 \pm 0.01$ & $47.7 \pm 22.6$
\end{tabular}



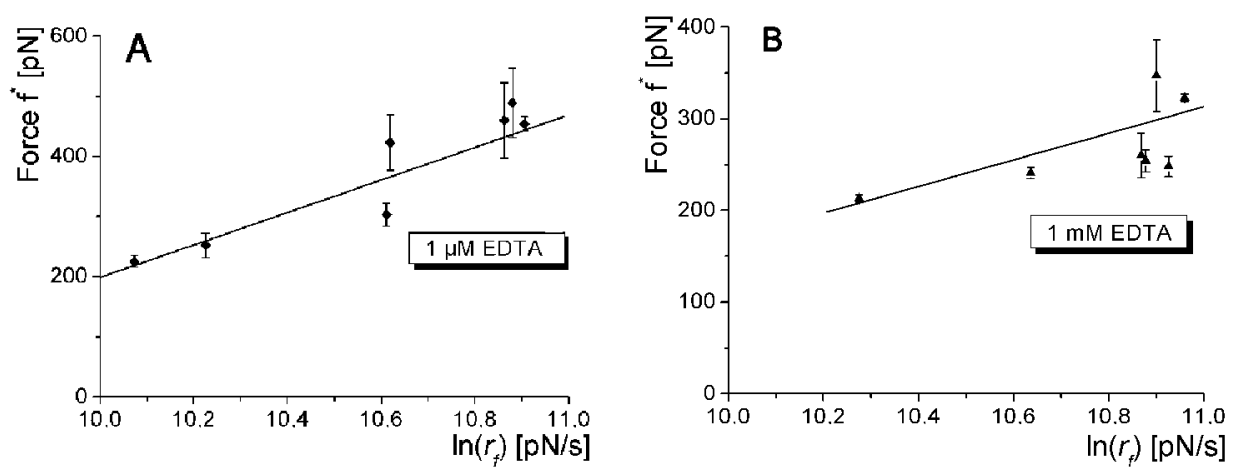

Fig. 6. The relation between the most probable unbinding force $f^{*}$ and the logarithm of the loading rate obtained for the BSA-EDTA pair in the presence of aqueous solutions of $1 \mu \mathrm{M}$ EDTA, and $1 \mathrm{mM}$ EDTA shown in the upper (A) and lower (B) part, respectively.

(at position of $r_{0}=0.15 \AA$ and $r_{0}=0.28 \AA$ for low and high EDTA concentration, respectively). Such significant increase (87\%) in the position of the energy barrier might be attributed to the influence of the free EDTA molecules on the dissociation process. However, the presence of EDTA did not influence on the dissociation rates that are related to the bond lifetimes. Their values remained roughly the same (39.5 s-1 and $47.7 \mathrm{~s}^{-1}$ for low and high concentration, correspondingly). The standard t-test $(0.05$ level $)$ confirmed that there was no significant differences between rates for $1 \mathrm{mM}$ and $1 \mu \mathrm{M}$ concentrations of EDTA. These results show that the EDTA molecules present in the solution may alter the dissociation kinetics. The calculated Bell model parameters for the studied pair of molecules are in the same range as those obtained in Ref. [15] for other molecules of the ligand-receptor pairs. However, it should be pointed out that the determination of the position of the energy barrier was more precise (errors were much smaller) than the estimation of the dissociation rates, where the calculated maximum errors exceeded sometimes $70 \%$.

\section{Conclusions}

The atomic force microscope was applied to study the interaction forces between albumin and ethylene(di)aminetetraacetic acid. The relationship of the force depended linearly on the logarithm of the loading rate and showed the same relationship as the previously studied ligand-receptor interaction $[5,6,9]$. The parameters describing the dissociation process were estimated. They showed that in the case of the deionized water the dissociation was more rapid than in the case when free EDTA molecules were present.

Therefore, the AFM demonstrated the feasibility in the investigation of the dissociation processes not only in complexes where the molecular interaction is governed by the specific forces but also in cases where non-specific forces occur. 


\section{Acknowledgment}

This work was partially supported by the grant No. 6 P05A 12921 of the State Committee for Scientific Research.

\section{References}

[1] Handbook of Biological Physics, Eds. R. Lipowsky, E. Sackmann, Elsevier Science B.V., San Diego 1995, p. XXX.

[2] Ch. Zhu, J. Biomech. 33, 23 (2000).

[3] I. Schumakovitch, W. Grange, T. Strunz, P. Bertoncini, Biophys. J. 82, 517 (2002).

[4] P.J.L. Werten, H.W. Rémigy, B.L. de Groot, D. Fodias, A. Philipsen, H. Stahlberg, H. Grubmüller, A. Engel, FEBS Lett. 529, 65 (2002).

[5] E. Florin, V.T. Moy, H. Gaub, Science 264, 415 (1994).

[6] L. Chtcheglova, G. Dietler, Acta Phys. Pol. A 104, 321 (2003).

[7] E. van der Wegte, G. Hadziioannou, Langmuir 13, 4357 (1997).

[8] J. Zlatanova, S.M. Lindsay, S.H. Leuba, Progress in Biophysics and Molecular Biology 74, 37 (2000).

[9] Y.S. Lo, Y. Zhu, T. Beebe, Langmuir 17, 3741 (2001).

[10] S. Allen, X. Chen, J. Davies, A.C. Dawkes, J.C. Edwards, C.J. Roberts, J. Sefton, S.J.B. Tendler, P.M. Williams, Biochemistry 36, 7457 (1997).

[11] G. Bell, Science 200, 618 (1978).

[12] E. Evans, K. Ritchie, Biophys. J. 76, 2439 (1999).

[13] M. Guthold, R. Superfine, R.M. Taylor, Biomed. Microdev. 3, 9 (2001).

[14] D.J.F. Tees, R.E. Waugh, D.A. Hammer, Biophys. J. 80, 668 (2001).

[15] E. Evans, Biophys. Chem. 82, 83 (1999).

[16] A. Vinckier, P. Gervasoni, F. Zaugg, U. Ziegler, P. Lindner, P. Groscurth, A. Plückthun, G. Semenza, Biophys. J. 74, 3256 (1998).

[17] X.M. He, D.C. Carter, Nature 358, 209 (1992).

[18] C. Ladaviere, T. Delair, A. Domard, C. Pichot, B. Mandrand, J. Appl. Polymer Sci. 72, 1565 (1999).

[19] M. Lekka, J. Lekki, A.P. Shoulyarenko, B. Cleff, Z. Stachura, Polish J. Pathol. 47, 51 (1996).

[20] R. De Paris, T. Strunz, K. Oroszlan, H.J. Güntherodt, M. Hegner, Single Molecules 1, $285(2000)$.

[21] R. Merkel, P. Nassoy, A. Leung, K. Ritchie, E. Evans, Nature 397, 50 (1999).

[22] T. Strunz, K. Oroszlan, I. Schumakovitch, H.J. Güntherodt, M. Hegner, Biophys. J. 9, 206 (2000). 